\title{
MR Imaging of Myeloperoxidase Activity in a Model of the Inflamed Aneurysm Wall
}

\author{
M.J. Gounis, I.M.J. van der Bom, A.K. Wakhloo, S. Zheng, J.-Y. Chueh, A.L. Kühn, and A.A. Bogdanov Jr
}

\begin{abstract}
BACKGROUND AND PURPOSE: Although myeloperoxidase activity in vivo can be visualized by using noninvasive imaging, successful clinical translation requires further optimization of the imaging approach. We report a motion-sensitized driven-equilibrium MR imaging approach for the detection of a myeloperoxidase activity-specific gadolinium-containing imaging agent in experimental aneurysm models, which compensates for irregular blood flow, enabling vascular wall imaging in the aneurysm.
\end{abstract}

MATERIALS AND METHODS: A phantom was built from rotational angiography of a rabbit elastase aneurysm model and was connected to a cardiac pulse duplicator mimicking rabbit-specific flow conditions. A Tl-weighted turbo spin-echo-based motion-sensitized drivenequilibrium pulse sequence was optimized in vitro, including the addition of fat suppression and the selection of the velocity-encoding gradient parameter. The optimized sequence was applied in vivo to rabbit aneurysm models with and without inflammation in the aneurysmal wall. Under each condition, the aneurysms were imaged before and after intravenous administration of the imaging agent. The signal-to-noise ratio of each MR imaging section through the aneurysm was calculated.

RESULTS: The motion-sensitized driven-equilibrium sequence was optimized to reduce flow signal, enabling detection of the myeloperoxidase imaging agent in the phantom. The optimized imaging protocol in the rabbit model of saccular aneurysms revealed a significant increase in the change of SNR from pre- to post-contrast MR imaging in the inflamed aneurysms compared with naïve aneurysms and the adjacent carotid artery $(P<.0001)$.

CONCLUSIONS: A diagnostic MR imaging protocol was optimized for molecular imaging of a myeloperoxidase-specific molecular imaging agent in an animal model of inflamed brain aneurysms.

ABBREVIATIONS: LPS = lipopolysaccharide; MPO = myeloperoxidase; MSDE = motion-sensitized driven-equilibrium; PHASES = Population, Hypertension, Age, Size of Aneurysm, Earlier Subarachnoid Hemorrhage from Another Aneurysm, and Site of Aneurysm; SPIR = spectral presaturation inversion recovery; UIA = unruptured intracranial aneurysms; VENC = velocity-encoding gradient parameter

D ue to the continuing improvements to the safety of unruptured intracranial aneurysm (UIA) treatment, there has been a steady increase in procedure numbers. Between 2001 and 2006, there was a 22-fold increase in UIAs treated by endovascular coil embolization, ${ }^{1}$ and in 2008, approximately 57,000 UIAs were ei-

Received April 10, 2014; accepted after revision June 30.

From the Department of Radiology (M.J.G., I.M.J.v.d.B., A.K.W., S.Z., J.-Y.C., A.L.K., A.A.B.), New England Center for Stroke Research; and Departments of Neurosurgery and Neurology (A.K.W.) and Radiology (A.A.B.), Laboratory of Molecular Imaging Probes, University of Massachusetts Medical School, Worcester, Massachusetts.

This work was funded by the National Institute on Aging grant 5R01AG034901-02. Paper previously presented at: Annual Meeting of the International Society for Magnetic Resonance in Medicine, April 20-26 2013; Salt Lake City, Utah; American Society of Mechanical Engineering Summer Bioengineering Conference, June 2629, 2013; Sunriver, Oregon; and Society of Neurolnterventional Surgery Conference, July 29 to August 1, 2013; Miami, Florida.

Please address correspondence to Matthew J. Gounis, PhD, Department of Radiology, New England Center for Stroke Research, University of Massachusetts ther surgically clipped or coiled in the United States alone. ${ }^{2}$ While the annual risk of rupture of an UIA is estimated to be between $0 \%$ and $33 \%$ depending on size, morphology, anatomic location, and history of subarachnoid hemorrhage, ${ }^{3,4}$ the combined rate of morbidity and mortality resulting from coil embolization and surgical clipping is approximately $5 \%$ and $14 \%$, respectively. ${ }^{2}$ Thus, the risk-benefit analysis does not support interventional treatment of all unruptured aneurysms but dictates, rather, that UIAs be carefully selected on the basis of their relative risk of rupture. Currently, it is not possible to accurately assess the UIA risk of rupture. The challenge therefore lies in the ability to positively identify UIAs that are progressing to rupture. Accordingly, new methods for improving this capability will be critical for im-

Medical School, 5 Lake Ave N, SA-107R, Worcester, MA 01655; e-mail: Matthew. Gounis@umassmed.edu

- Indicates open access to non-subscribers at www.ajnr.org

http://dx.doi.org/10.3174/ajnr.A4135 
proving clinical decision-making in managing individual cases. Toward this end, a diagnostic method has been recently devised to enable imaging of UIA wall inflammation by using iron oxide nanoparticles. ${ }^{5,6}$

The recent focus on inflammation-induced events and markers is primarily due to their role as a potential contributor to aneurysm rupture (reviewed in Tulamo et $\mathrm{al}^{7}$ ). Inflammation is likely associated with vascular remodeling which, with time, exposes the aneurysm to increased risk of rupture. One of the major inflammation-associated tissue markers, myeloperoxidase (MPO), is a secretable heme-containing oxidoreductase of azurophilic granules of polymorphonuclear cells. Secreted MPO produces chlorinating bactericidal species (eg, hypochlorous acid) from hydrogen peroxide during neutrophilic respiratory burst and chloride anions. In addition to a well-known role in the host defense system against microorganisms, MPO has been implicated in the initiation and destabilization of atherosclerotic plaques (reviewed in Nicholls and $\mathrm{Hazen}^{8}$ ). Recently, MPO presence in human brain aneurysm specimens was shown to correlate with the Population, Hypertension, Age, Size of Aneurysm, Earlier Subarachnoid Hemorrhage from Another Aneurysm, and Site of Aneurysm (PHASES) model $^{9}$ of aneurysm risk of rupture. ${ }^{10}$ Enzymatic activity of neutrophilic myeloperoxidase can be used as a highly selective and sensitive target for detecting inflammation by using clinical imaging modalities (MR imaging, SPECT). ${ }^{11-15}$ A gadolinium (III) chelate MPO activity-sensing MR imaging probe has also been investigated in an animal model of inflamed saccular aneurysms. ${ }^{16}$ With a 3D T1-weighted fast-field echo pulse sequence, local accumulation of the probe as determined by enhancement correlated with MPO activity at the site of Escherichia coli lipopolysaccharide (LPS) injection. Signal changes measured on MR imaging were possible at the clinical field strength (3T) and gadolinium (III) dose.

Although these results are promising, the MR imaging protocols (specifically for axial black-blood imaging) have to be optimized to detect the presence of MPO within an aneurysm, especially given the complexity of intra-aneurysmal flow and the resultant attenuation of the MR imaging signal. At this juncture, the MPO activity-specific imaging agent is experimental and is not approved for human use. Therefore, we optimized the MR imaging protocol for detecting MPO activity in both in vitro and in vivo aneurysm models.

\section{MATERIALS AND METHODS}

\section{In Vitro MR Imaging Sequence Optimization}

All MR imaging studies were conducted on a 3T Achieva scanner (Philips Healthcare, Best, the Netherlands) by using an 8-element, receive-only knee coil (Philips Healthcare). Radiofrequency was transmitted with the built-in quadrature body coil. To optimize MR imaging sequences that are insensitive to the complex intraaneurysmal flow patterns, we first conducted an in vitro phantom study. A silicone vascular replica was built from 3D rotational angiography of a rabbit elastase aneurysm by using a rapid prototyping technique previously described. ${ }^{17}$ The phantom was connected to a flow-loop-driven programmable pulsatile pump simulating the aortic waveform of the rabbit $^{18}$ by using a blood analog working fluid that matched the T1 and viscosity of blood. We applied a turbo spin-echo pulse sequence combined with the following components to suppress blood flow signal: black-blood double inversion recovery, ${ }^{19}$ small FOV quadrature inversion recovery, ${ }^{20}$ and motion-sensitized driven-equilibrium (MSDE). ${ }^{21}$ All images were acquired with 2-mm sections along the long axis of the aneurysm in a $100-\mathrm{mm}$ FOV with an acquired in-plane resolution of $0.4 \times 0.4 \mathrm{~mm}$. The TSE-based double inversion recovery, small FOV quadrature inversion recovery, and MSDE scan parameters included TR/TE of $456 / 10 \mathrm{~ms}, 750 / 11 \mathrm{~ms}$, and $698 / 9 \mathrm{~ms}$; and flip angles of $90^{\circ}, 90^{\circ}$, and $70^{\circ}$, respectively. The number of signal averages for all sequences was 2 . The flow velocity-encoding gradient parameter (VENC) had values between 1 and $8 \mathrm{~cm} / \mathrm{s}$.

Once the flow was adequately suppressed, the phantom aneurysm was embedded in solid coconut oil to account for extravascular fat. Images were also acquired using a spectral presaturation inversion recovery (SPIR) fat-suppression method. Finally, a small capsule containing $150 \mu \mathrm{mol} / \mathrm{L}$ of Gd-DTPA in a volume of $\sim 30 \mu \mathrm{L}$ was included in the aneurysm replica to determine whether sufficient flow suppression was achieved to enable detection of a representative amount of retained Gd-containing imaging agent.

Additional sequences included the following: a 3D time-offlight sequence (voxel size, $0.208 \times 0.208 \times 1 \mathrm{~mm}^{3}$; gap, -0.5 $\mathrm{mm}$; TR/TE, $20.1 / 3.6 \mathrm{~ms}$; flip angle, $20^{\circ}$; number of signal averages, 2), and a coronal and axial T1-weighted fast-field echo sequence (voxel size, $0.625 \times 0.625 \times 2 \mathrm{~mm}^{3}$; gap, $-1.0 \mathrm{~mm}$; TR/ TE, 17.9/2.3 ms; flip angle, $25^{\circ}$ ).

\section{In Vivo MR Imaging of a Rabbit Model}

All in vivo procedures were first approved by our Institutional Animal Care and Use Committee. A saccular aneurysm model in New Zealand White rabbits $(n=8)$ was created as previously described. ${ }^{22}$ Three weeks after the aneurysms were created, the animals were re-anesthetized and imaged by using the MR imaging protocol established from our in vitro phantom study. Due to the location of the aneurysm, respiratory gating was used. The naïve aneurysms were imaged before and 3 hours after IV administration of the MPO activity-specific paramagnetic imaging agent (DTPA[Gd] bis-hydroxytryptamide, bis-5HT-DTPA[Gd]) at a dose of $0.1 \mathrm{mmol} \mathrm{Gd} / \mathrm{kg}$ in $15 \mathrm{~mL}$ of $5 \%$ meglumine, $\mathrm{pH} 7{ }^{16}$ The synthesis, purification, and in vitro testing of bis-5HTDTPA $(\mathrm{Gd})$ were essentially performed as previously reported. ${ }^{14}$

One week after the baseline MR imaging study was performed, 7 animals were returned to the surgical suite for LPS-induced inflammation of the aneurysm wall, as previously described. ${ }^{16}$ Briefly, with the animal under general anesthesia, a $6 \mathrm{~F}$ guide catheter was positioned at the root of the brachiocephalic artery by using a standard transfemoral approach. Thereafter, a standard microcatheter (0.016-inch inner diameter) was navigated into the aneurysm sac over a 0.014 -inch guidewire. With the microcatheter placed deep into the dome, a 0.014-inch stainless steel hypotube with a ground distal tip was advanced through the microcatheter and inserted carefully into the aneurysm wall. LPS (1-mg, $200-\mu \mathrm{L}$ total volume) was injected through the hypotube for approximately 10 minutes. The remaining animal served as a nega- 


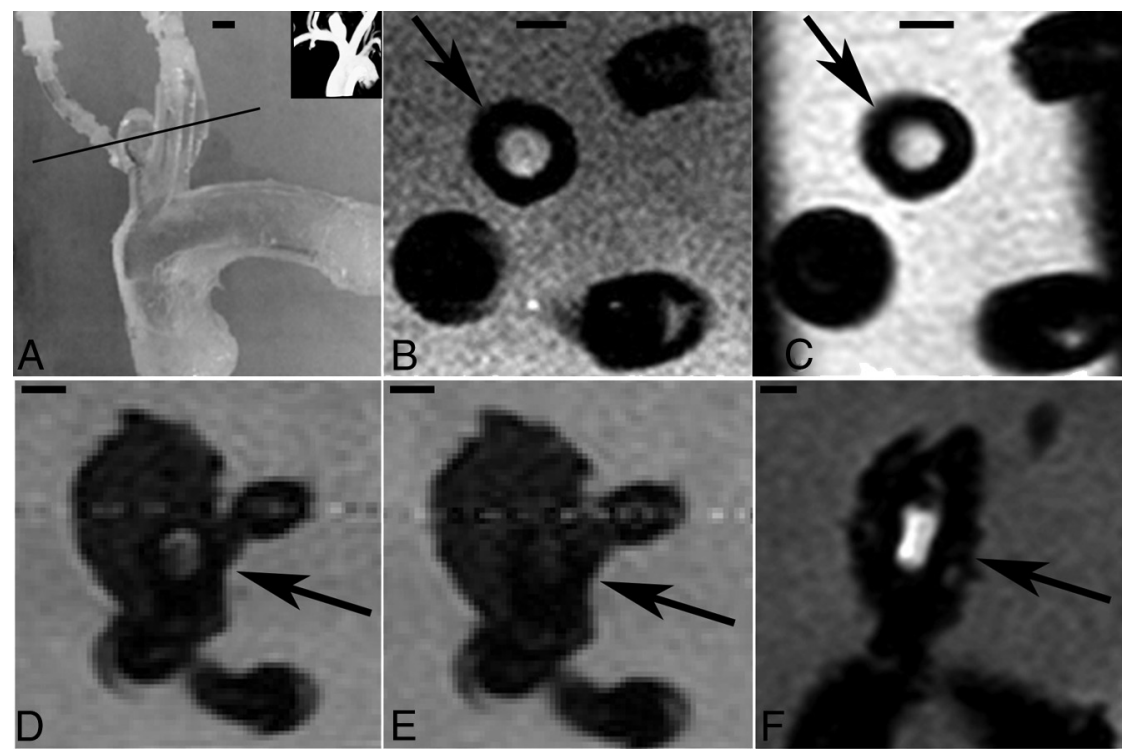

FIG 1. A, A silicone replica of a rabbit elastase-induced aneurysm of the right common carotid artery (arrows) was built and subjected to MR imaging (axial projections along a line in $A$; the inset is rotational angiography data on which the model was built). Double inversion recovery black-blood $(B)$ and small FOV quadrature recovery $(C)$ suppressed the flow signal in the adjacent arteries but not within the aneurysm dome (arrows). The MSDE sequence combined with SPIR to suppress the surrounding fat with a VENC of $2 \mathrm{~cm} / \mathrm{s}$ had persistent flow signal in the aneurysm dome ( $D$, arrow), which was eliminated with a VENC of $1 \mathrm{~cm} / \mathrm{s}(E)$. With a VENC of 1 $\mathrm{cm} / \mathrm{s}$ and SPIR for fat suppression, we were able to image a realistic Gd-DTPA contrast agent concentration (150 $\mu \mathrm{mol} / \mathrm{L})$ contained within a capsule implanted in the aneurysm ( $F$, frontal projection). Horizontal scale bars represent $3 \mathrm{~mm}$.

tive control. Two days after the LPS administration, the MR imaging study was repeated before and after intravenous infusion of the MPO activity-specific imaging agent. After the final imaging study, the animals were euthanized and the aneurysms were surgically explanted for histologic analysis. The aneurysm tissue was embedded in tissue-freezing medium, snap-frozen in liquid nitrogen, and stored at $-80^{\circ} \mathrm{C}$ until processing.

Rabbit tissues were carefully divided in half along the long axis of the aneurysm. The first section was processed by using an alkaline phosphatase immunohistochemistry approach due to the high autofluorescence of rabbit sections. The rabbit aneurysm and contralateral carotid artery sample sections were incubated for 90 minutes in 50- $\mathrm{mmol} / \mathrm{L}$ tris, $100-\mathrm{mmol} / \mathrm{L} \mathrm{NaCl}$ (tris-buffered saline), containing $1-\mathrm{mmol} / \mathrm{L}$ ethylenediaminetetraacetic acid, $\mathrm{pH} 7$, to block endogenous phosphatase activity, and were blocked in $5 \%$ bovine serum, $10-\mathrm{mg} / \mathrm{mL}$ bovine serum albumin, tris-buffered saline, $\mathrm{pH} 7.5$, for 2 hours. The primary antibodies (cross-reacting with rabbit antigens) were the corresponding mouse anti-human monoclonal antibodies (2C7, dilution: 1:100; Abcam, Cambridge, Massachusetts). The secondary antibody (anti-mouse rabbit-alkaline phosphatase conjugate; Roche, Basel, Switzerland) was used at 1:1000 dilution.

The remaining aneurysm tissue was subjected to an analysis of MPO activity performed by using a commercially available MPO kit (Fluoro MPO; Cell Technology, Mountain View, California). A typical assay included homogenization of 1-10 mg of salinerinsed, rapidly defrosted tissue in a vial containing a slurry of 300 - $\mathrm{mg}$ sterile glass beads ( 1 -mm diameter) suspended in $0.5 \mathrm{~mL}$ of $0.5 \%$ solution of hexadecyltrimethylammonium bromide, 10 $\mathrm{mmol} / \mathrm{L} \mathrm{N}$-ethylmaleimide in $0.1-\mathrm{mol} / \mathrm{L}$ potassium phosphate,
$\mathrm{pH}$ 6.5. The homogenization was performed by using a mini-BeadBeater (BioSpec Products, Bartlesville, Oklahoma) for 12 cycles (30 seconds each) with 1-minute cooling on ice between the cycles. The final disruption was performed by using 3 freeze-thaw cycles. The samples were cleared by centrifugation (8000xg, 5 minutes), and the activity of MPO was determined in the supernatant by using a fluorescent analog of MPO substrate in the presence of hydrogen peroxide. The rates of fluorescence increase were determined by using a kinetic assay $(\lambda \mathrm{ex}=550$ $\mathrm{nm}, \lambda$ em $=600 \mathrm{~nm})$ with an MPO standard solution (Cell Technology) to generate calibration curves. The protein content in the homogenized samples was determined by using a bicinchoninic acid kit (Bio-Rad Laboratories, Berkeley, California). The total MPO activity was expressed in international units per milligram wet sample and international units per milligram extractable protein.

\section{MR Image and Statistical Analysis}

Images from the phantom study were qualitatively reviewed by the entire investigative team (all authors) for flow and fat suppression and sufficient contrast-to-noise to distinguish the Gd-DTPA capsule from the background. Selection of the optimized sequence in vitro was reached by consensus of the team. In the rabbit study, 2 experienced observers (M.J.G., 10 years; A.L.K., 2 years) created elliptic regions of interest around the aneurysm and left common carotid artery. Region-of-interest selection was reviewed by a neuroradiologist with $>20$ years of experience in cerebrovascular imaging (A.K.W.). The signal-to-noise ratio was calculated by dividing the mean intensity within the region of interest by the SD of the image background. The SNR pre-contrast was subtracted from the postcontrast value, and the change in SNR was compared between the naïve and inflamed aneurysms and the left common carotid artery.

The results are expressed as mean \pm standard error of the mean. A repeated-measures ANOVA with a Tukey multiple comparisons posttest was used to make comparisons of the SNR changes. $P<.05$ was statistically significant.

\section{RESULTS}

\section{In Vitro MR Imaging Sequence Optimization}

The vascular replica used for sequence optimization is shown in Fig $1 A$. Although excellent flow suppression was achieved with the double inversion recovery and small FOV quadrature inversion recovery techniques in the adjacent arteries, substantial flow signal within the aneurysm persisted (Fig $1 B,-C$ ). The MSDE technique by using a VENC of $1 \mathrm{~cm} / \mathrm{s}$ was shown to provide excellent flow suppression compared with larger VENC values (Fig 1D, -E). We confirmed that the MSDE sequence with a VENC of $1 \mathrm{~cm} / \mathrm{s}$ and the addition of SPIR eliminated the signal from blood flow 


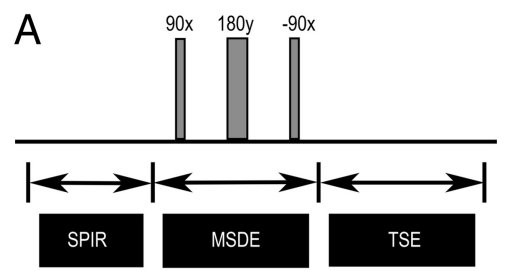

B

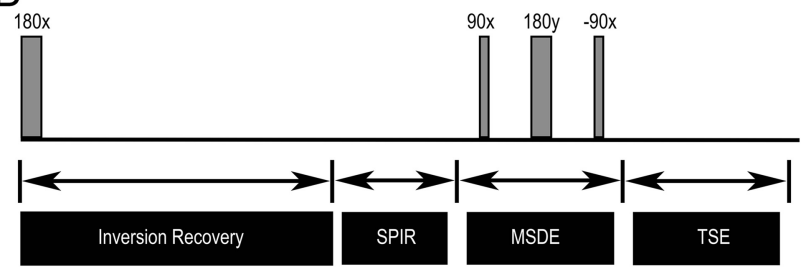

FIG 2. Schematic of the TSE-based MSDE pulse sequence optimized in the in vitro study $(A)$, subsequently modified for in vivo rabbit aneurysm imaging $(B)$.

and adjacent fat, respectively, yet provided sufficient T1-weighted contrast to image the capsule containing $0.1-\mathrm{mmol} / \mathrm{L}$ Gd-DTPA inserted and affixed in the aneurysm dome (Fig $1 F$ ). The optimal sequence was the fat-suppressed T1-weighted TSE-based MSDE pulse sequence (Fig 2A), including the following parameters: TR/TE 698/9 ms; flip angle, 70; TSE factor, 4 with low-high profile order; VENC, $1 \mathrm{~cm} / \mathrm{s}$; and number of signal averages, 2 .

\section{In Vivo MR Imaging of a Rabbit Model}

We transferred the optimized MSDE sequence to the rabbit aneurysm model. However, due to the location of the aneurysm, significant motion artifacts due to respiration were observed. Therefore, MR imaging was performed with respiratory triggering. Data acquisition was performed during the expiration phase by using a multi-2D method that required $\mathrm{TR}=5000 \mathrm{~ms}$ as determined by the length of the respiratory cycle. Consequently, T1-weighting for contrast enhancement was lost. To overcome this loss, we added an inversion pulse to

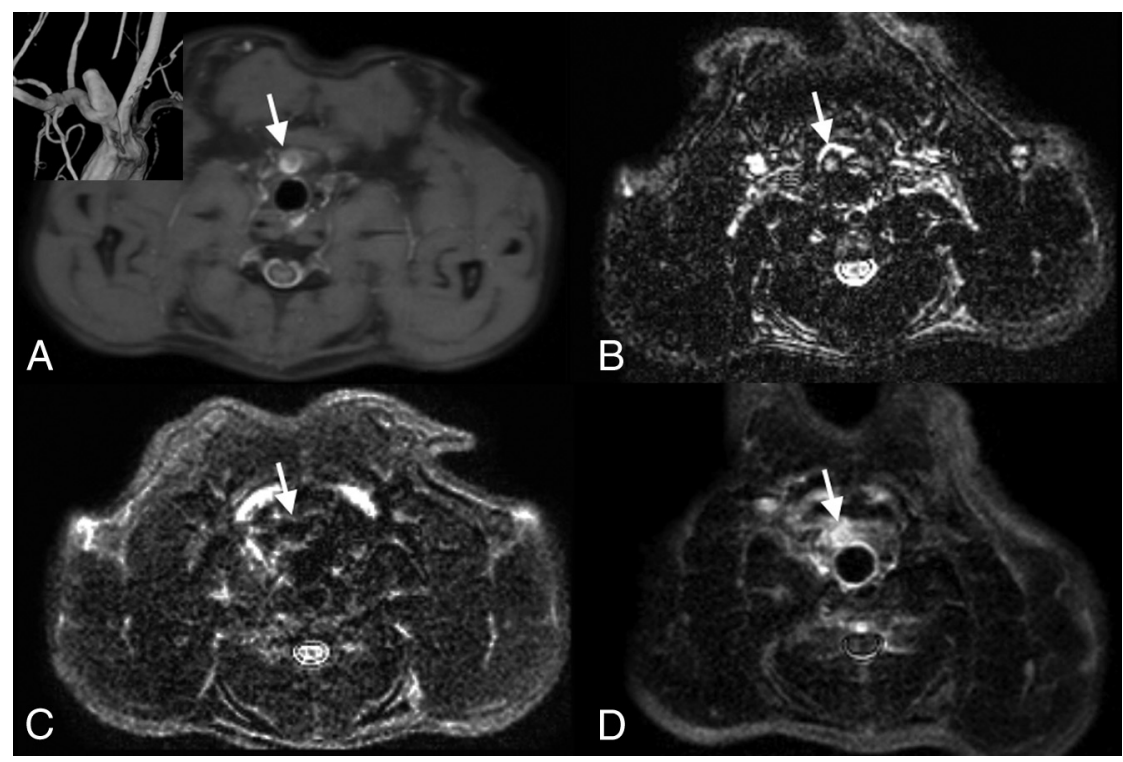

FIG 3. Model of a saccular aneurysm (arrows) in the rabbit ( $A$, axial projection, turbo spin-echo with SPIR, inset rotational angiogram in frontal oblique projection). MSDE images before receiving the MPO-specific contrast agent (B) and after IV administration of bis-5HT-DTPA(Gd) in the naïve $(C)$ and inflamed $(D)$ aneurysms $(0.1-\mathrm{mmol} \mathrm{Gd} / \mathrm{kg})$.
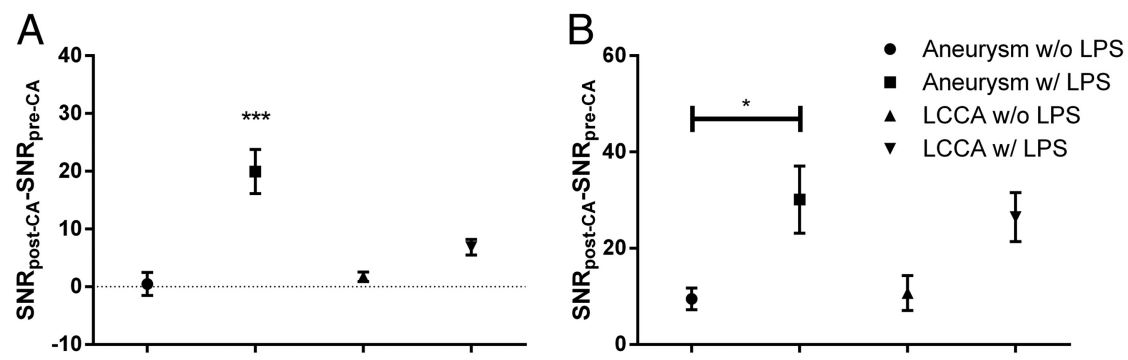

FIG 4. A, Signal-to-noise ratio change of the inflamed aneurysm was highly significant by using the MSDE sequence and was negligible in the naïve aneurysm as well at the left common carotid artery, suggesting adequate flow suppression. $B$, SNR change calculated from the T1-fast-field echo axial sections shows a significant increase between the inflamed and naive aneurysm model, but no differences between the inflamed aneurysm and the left common carotid artery either before or after LPS administration, suggesting flow dependence. The asterisk indicates $P<.05$; triple asterisks, $P<.0001$, contrast agent: bis-5HT-DTPA(Gd). the sequence to gain $\mathrm{T} 1$ sensitivity by inversion recovery (Fig $2 B$ ). The inversion recovery delay time $(800 \mathrm{~ms})$ was determined by minimizing the signal generated by the aneurysm wall in an effort to maximize the signal difference with respect to post-5HT-DTPA $(\mathrm{Gd})$ imaging (Fig 3). A TSE method with a TSE factor of 16 was used for imaging, and a low-to-high profile was used to achieve a small TE value of $8.6 \mathrm{~ms}$.

Compared with the naïve aneurysm, on average, a 40 -fold increase in the SNR change from pre- to post-contrast MSDE imaging in the inflamed aneurysm model was found (Fig 4A). The mean SNR change in the naïve left common carotid artery and the left common carotid artery following injection of LPS into the aneurysm wall was 1.7 and 6.8 , respectively. The model of the inflamed aneurysm had a significant increase in the SNR change compared with all controls $(P<.0001)$. Similar trends in the SNR change were observed by using the 3D T1-weighted fast-field echo sequence; however, significant differences between the inflamed aneurysm versus the control left common carotid artery were not found (Fig 4B). The aneurysm model including the LPS modification had 19.4 \pm 10.8 U MPO per milligram, whereas the negative control animal had $0.45 \mathrm{U}$ MPO per milligram. Similar to that in our previous study, ${ }^{16}$ the LPSinduced inflammation demonstrated a large infiltration of MPO and macrophages within the aneurysm wall compared with the naïve control (Fig 5). 


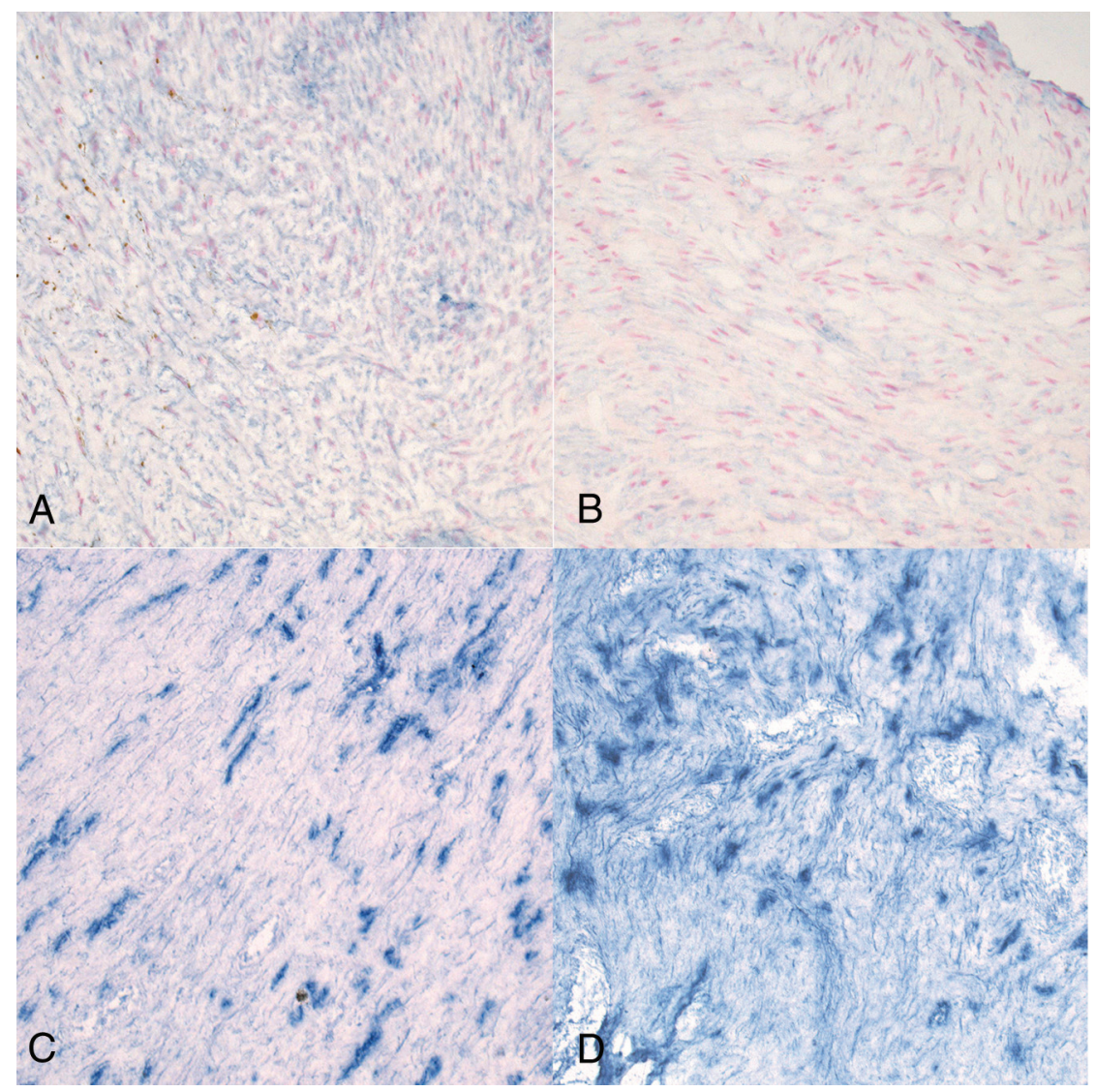

FIG 5. Histology of the aneurysm wall in the animal model without $(A$ and $B)$ and with $(C$ and $D)$ perivascular injection with LPS. The naïve aneurysm has limited macrophage infiltration $(A)$ and no MPO $(B)$. The modification with LPS induces significant macrophage $(C)$ infiltration and has prominent distribution of MPO $(D)$. All images are at $\times 20$ magnification.

as cytokines, as well as MPO and elastase ${ }^{30}$ which reduce wall strength and thus allow the aneurysm to expand.

The direct role of neutrophil-derived MPO in UIA rupture has not been extensively studied, but a confluence of evidence from research aimed at determining the risk of acute cardiovascular events identified MPO as a key player (reviewed in Schindhelm et $\mathrm{al}^{31}$ ). More recently, MPO presence has been correlated with the PHASES model of the annual rupture risk of aneurysms. ${ }^{10}$ In addition to its role in inflammation, MPO mediates oxidative cell stress due to the production of reactive oxygen species contributing to vessel wall damage. Building on previous work with human aneurysm tissue, ${ }^{32,33}$ a rat model was developed in which an allogeneic arterial graft was decelluarized before its anastomosis to the aorta of the experimental animal. ${ }^{34}$ Nearly $50 \%$ of these decelluarized aneurysms progressed to rupture, whereas control aneurysms remained stable. Histologic analysis demonstrated that thrombus formation within the decelluarized aneurysm could not organize; this feature led to recanalization, inflammation, wall degeneration, and ultimate rupture. Similar conclusions regarding the role of incomplete thrombosis and subsequent inflammatory cell infiltration into the aneurysm wall with

\section{DISCUSSION}

With advances in noninvasive neuroimaging, aneurysms are being detected incidentally with increasing frequency. ${ }^{23,24}$ The patients diagnosed with UIA and facing the potentially dismal outcome of aneurysmal SAH (associated with a 50\% mortality rate) experience a significant decrease in quality of life. ${ }^{25}$ An objective test to assess rupture risk could potentially impact the decision to treat UIAs. To achieve this goal in clinical practice, thorough evaluation of multiple sequences may lead to a unified MR imaging protocol for risk stratification of intracranial aneurysms.

We have proposed to exploit the relationship between inflammation and aneurysm progression to rupture (reviewed in Tulamo et $\mathrm{al}^{7}$ ) as a means of assessing rupture risk through MR imaging. The exact time course of aneurysm progression to rupture has not been determined in humans. Most of our understanding of factors that may contribute to aneurysm progression and rupture has been gained through studies using animal models. The consensus from these studies is that aneurysm growth is strongly influenced by mediators of inflammation such as interleukin-1- $\beta,{ }^{26}$ nuclear factor $\kappa \mathrm{B},{ }^{27}$ and monocyte chemoattractant protein-1. ${ }^{28}$ The presence of these molecules in the vessel wall is most likely due to leukocyte infiltration, which has recently been shown to have a direct link to aneurysm development. ${ }^{29}$ Neutrophils are the most abundant leukocytes capable of trafficking into the vessel wall. They secrete an array of signaling molecules such marked degeneration were confirmed in a swine venous pouch aneurysm model. ${ }^{35}$

To enable molecular imaging of MPO activity with high resolution, we selected the realistic rabbit elastase model of saccular aneurysms due to the complex intra-aneurysmal hemodynamics, which are similar to that of human intracranial aneurysms. ${ }^{36}$ The TSE-based MSDE sequence in a phantom model allowed suppression of the signal from intra-aneurysmal blood flow to a degree that was not achievable by using double inversion recovery ${ }^{37}$ or small FOV quadrature inversion recovery ${ }^{20}$ black-blood imaging. The optimized sequence demonstrated a 40-fold increase in SNR in the inflamed aneurysm model compared with the naive aneurysm following 5HT-DTPA(Gd) administration. This is a dramatic improvement from the previously used T1-fast-field echo protocol. ${ }^{16}$ Contrast retention within the aneurysm wall has been observed for up to 6 hours, ${ }^{16}$ and in other models, clearance of the contrast has been documented at 24 hours. ${ }^{15}$ Further study of contrast dynamics as these apply to aneurysms is needed.

The MPO activity of the aneurysm model after being treated with LPS was similar to that measured in unruptured human aneurysms; however, the negative control naïve rabbit aneurysm had $>40$-fold reduction in MPO activity. ${ }^{10}$ A limitation of our approach is that direct comparisons of the enzymatic MPO activity measurements in human specimens and the rabbit model are 
not possible due to the well-known interspecies variability. ${ }^{38}$ Qualitatively, the alkaline phosphatase staining showed an abundance of MPO in the inflamed rabbit aneurysm (Fig 4). Another limitation of this model is the artificial induction of inflammatory changes that do not involve hemodynamic activation of the endothelium and subsequent aneurysm wall remodeling, which is suspected in aneurysm pathogenesis. Thoughtful research has focused on characterizing intra-aneurysmal hemodynamics associated with rupture, ${ }^{39-41}$ and correlating local hemodynamics with noninvasive imaging of MPO activity presents an exciting experimental paradigm. Although the animal model of inflamed intracranial aneurysm presented herein may not be relevant to achieve this goal, it may be possible to link intra-aneurysmal hemodynamics with MPO activity in clinical aneurysm specimens obtained during clipping. ${ }^{10}$

\section{CONCLUSIONS}

A diagnostic MR imaging protocol has been optimized for detection of an MPO-specific paramagnetic imaging agent in an animal model of inflamed intracranial aneurysms.

Disclosures: Matthew J. Gounis—RELATED: Grant: Philips Healthcare, ${ }^{\star}$ Comments: Research instrumentation and science keys were provided by Philips Healthcare; UNRELATED: Consultancy: Stryker Neurovascular, Surpass Inc (contract research organization), Surpass Medical, Codman Neurovascular, Comments: fee-per-hour consulting; Grants/Grants Pending: National Institutes of Health, ${ }^{*}$ Stryker Neurovascular, ${ }^{*}$ Codman Neurovascular, ${ }^{*}$ Lazarus Effect, ${ }^{*}$ Covidien, ev3, ${ }^{*}$ Fraunhofer Institute, ${ }^{\star}$ Philips Healthcare, ${ }^{\star}$ Silk Road, ${ }^{*}$ Tay Sachs Foundation, ${ }^{\star}$ Lundbeck. ${ }^{\star}$ Imramsjah Martijn John van der Bom—UNRELATED: Employment: Philips Healthcare. Ajay K. Wakhloo-UNRELATED: Consultancy: Stryker Neurovascular; Employment: Surpass Medical; Grants/Grants Pending: Philips Healthcare*; Payment for Lectures (including service on Speakers Bureaus): Harvard Medical School, Miami Baptist Health; Stock/Stock Options: Surpass Medical. Alexei A. Bogdanov Jr-RELATED: Grant: National Institute on Aging, 5R01AG034901-02. *Money paid to the institution.

\section{REFERENCES}

1. Alshekhlee A, Mehta S, Edgell RC, et al. Hospital mortality and complications of electively clipped or coiled unruptured intracranial aneurysm. Stroke 2010;41:1471-76

2. Brinjikji W, Rabinstein AA, Nasr DM, et al. Better outcomes with treatment by coiling relative to clipping of unruptured intracranial aneurysms in the United States, 2001-2008. AJNR Am J Neuroradiol 2011;32:1071-75

3. Morita A, Kirino T, Hashi K, for the UCAS Japan Investigators. The natural course of unruptured cerebral aneurysms in a Japanese cohort. N Engl J Med 2012;366:2474-82

4. Wiebers DO, Whisnant JP, Huston J, 3rd, et al. Unruptured intracranial aneurysms: natural history, clinical outcome, and risks of surgical and endovascular treatment. Lancet 2003;362:103-10

5. Hasan DM, Mahaney KB, Magnotta VA, et al. Macrophage imaging within human cerebral aneurysms wall using ferumoxytol-enhanced MRI: a pilot study. Arterioscler Thromb Vasc Biol 2012;32:1032-38

6. Hasan D, Chalouhi N, Jabour P, et al. Early change in ferumoxytolenhanced magnetic resonance imaging signal suggests unstable human cerebral aneurysm: a pilot study. Stroke 2012;43:3258-65

7. Tulamo R, Frösen J, Hemesniemi J, et al. Inflammatory changes in the aneurysm wall: a review. J Neurointervent Surg 2010;2:120-30

8. Nicholls SJ, Hazen SL. Myeloperoxidase and cardiovascular disease. Arterioscler Thromb Vasc Biol 2005;25:1102-11

9. Greving JP, Wermer MJ, Brown RD Jr, et al. Development of the PHASES score for prediction of risk of rupture of intracranial aneurysms: a pooled analysis of six prospective cohort studies. Lancet Neurol 2014;13:59-66

10. Gounis MJ, Vedantham S, Weaver JP, et al. Myeloperoxidase in hu- man intracranial aneurysms: preliminary evidence. Stroke 2014; 45:1474-77

11. Chen JW, Pham W, Weissleder R, et al. Human myeloperoxidase: a potential target for molecular MR imaging in atherosclerosis. Magn Reson Med 2004;52:1021-28

12. Chen JW, Querol Sans M, Bogdanov A Jr, et al. Imaging of myeloperoxidase in mice by using novel amplifiable paramagnetic substrates. Radiology 2006;240:473-81

13. Querol M, Chen JW, Weissleder R, et al. DTPA-bisamide-based MR sensor agents for peroxidase imaging. Org Lett 2005;7:1719-22

14. Querol M, Chen JW, Bogdanov AA Jr. A paramagnetic contrast agent with myeloperoxidase-sensing properties. Org Biomol Chem 2006;4:1887-95

15. Ronald JA, Chen JW, Chen Y, et al. Enzyme-sensitive magnetic resonance imaging targeting myeloperoxidase identifies active inflammation in experimental rabbit atherosclerotic plaques. Circulation 2009;120:592-99

16. DeLeo MJ 3rd, Gounis MJ, Hong B, et al. Carotid artery brain aneurysm model: in vivo molecular enzyme-specific MR imaging of active inflammation in a pilot study. Radiology 2009;252:696-703

17. Chueh JY, Wakhloo AK, Gounis MJ. Neurovascular modeling: small-batch manufacturing of silicone vascular replicas. AJNR Am J Neuroradiol 2009;30:1159-64

18. Seong J, Sadasivan C, Onizuka M, et al. Morphology of elastaseinduced cerebral aneurysm model in rabbit and rapid prototyping of elastomeric transparent replicas. Biorheology 2005;42:345-61

19. Park JK, Lee CS, Sim KB, et al. Imaging of the walls of saccular cerebral aneurysms with double inversion recovery black-blood sequence. J Magn Reson Imaging 2009;30:1179-83

20. Yarnykh VL, Yuan C. T1-insensitive flow suppression using quadruple inversion-recovery. Magn Reson Med 2002;48:899-905

21. Wang J, Yarnykh VL, Yuan C. Enhanced image quality in blackblood MRI using the improved motion-sensitized driven-equilibrium (iMSDE) sequence. J Magn Reson Imaging 2010;31:1256-63

22. Altes TA, Cloft HJ, Short JG, et al. 1999 ARRS Executive Council Award: creation of saccular aneurysms in the rabbit-a model suitable for testing endovascular devices. AJR Am J Roentgenol 2000;174:349-54

23. Tummala RP, Baskaya MK, Heros RC. Contemporary management of incidental intracranial aneurysms. Neurosurg Focus 2005; 18:E9-18

24. Vernooij MW, Ikram MA, Tanghe HL, et al. Incidental findings on brain MRI in the general population. $N$ Engl $J$ Med 2007;357:1821-28

25. Buijs JE, Greebe P, Rinkel GJE. Quality of life, anxiety and depression in patients with an unruptured intracranial aneurysm with or without aneurysm occlusion. Neurosurgery 2012;70:868-72

26. Moriwaki T, Takagi $\mathrm{Y}$, Sadamasa N, et al. Impaired progression of cerebral aneurysms in interleukin-1- $\boldsymbol{\beta}$-deficient mice. Stroke 2006;37:900-05

27. Aoki T, Kataoka H, Shimamura M, et al. NF- $\boldsymbol{\kappa} B$ is a key mediator of cerebral aneurysm formation. Circulation 2007;116:2830-40

28. Aoki T, Kataoka H, Ishibashi R, et al. Impact of monocyte chemoattractant protein-1 deficiency on cerebral aneurysm formation. Stroke 2009;40:942-51

29. Kanematsu Y, Kanematsu M, Kurihara C, et al. Critical roles of macrophages in the formation of intracranial aneurysm. Stroke 2011;42:173-78

30. Topham MK, Carveth HJ, McIntyre TM, et al. Human endothelial cells regulate polymorphonuclear leukocyte degranulation. FASEB J 1998;12:733-46

31. Schindhelm RK, van der Zwan LP, Teerlink T, et al. Myeloperoxidase: a useful biomarker for cardiovascular disease risk stratification? Clin Chem 2009;55:1462-70

32. Frösen J, Tulamo R, Heikura T, et al. Lipid accumulation, lipid oxidation, and low plasma levels of acquired antibodies against oxidized lipids associate with degeneration and rupture of the intracranial aneurysm wall. Acta Neuropathol Commun 2013;1:71 
33. Laaksamo E, Tulamo R, Liiman A, et al. Oxidative stress is associated with cell death, wall degradation, and increased risk of rupture of the intracranial aneurysm wall. Neurosurgery 2013;72:109-17

34. Marbacher S, Marjamaa J, Bradacova K, et al. Loss of mural cells leads to wall degeneration, aneurysm growth, and eventual rupture in a rat aneurysm model. Stroke 2014;45:248-54

35. Raymond J, Darsaut TE, Kotowski M, et al. Thrombosis heralding aneurysmal rupture: an exploration of potential mechanisms in a novel giant swine aneurysm model. AJNR Am J Neuroradiol 2013;34:346-53

36. Zeng Z, Kallmes DF, Durka MJ, et al. Hemodynamics and anatomy of elastase-induced rabbit aneurysm models: similarity to human cerebral aneurysms? AJNR Am J Neuroradiol 2011;32:595-601

37. Krings T, Mandell DM, Kiehl TR, et al. Intracranial aneurysms: from vessel wall pathology to therapeutic approach. Nat Rev Neurol 2011;7:547-59

38. Rausch PG, Moore TG. Granule enzymes of polymorphonuclear neutrophils: a phylogenetic comparison. Blood 1975;46:913-19

39. Cebral JR, Mut F, Weir J, et al. Quantitative characterization of the hemodynamic environment in ruptured and unruptured brain aneurysms. AJNR Am J Neuroradiol 2011;32:145-51

40. Valen-Sendstad K, Steinman DA. Mind the gap: impact of computational fluid dynamics solution strategy on prediction of intracranial aneurysm hemodynamics and rupture status indicators. $A J N R$ Am J Neuroradiol 2014;35:536-43

41. Pereira VM, Brina O, Bijlenga $P$, et al. Wall shear stress distribution of small aneurysms prone to rupture: a case-control study. Stroke 2014;45:261-64 